\title{
A NOTE ON A PAPER BY P. D. FINGH
}

\author{
D. H. ADAMS \\ (Received 21 November 1968) \\ Communicated by G. B. Preston
}

It is the purpose of this note to illustrate by example the resilts obtained in Finch [1]. All our definitions and notation are as in that paper, and we use them without reference. In his paper, Finch considered orthoposets and, in particular, orthomodular posets that had either the $A$ property or the $B$-property.

The question arises of the existence of orthomodular posets without these properties. We give an example of an orthomodular poset that has neither of these properties. In particular it is an example of an orthomodular poset whose completion by cuts is not orthomodular.

Consider the set $H=\{1,2,3,4,5,6\}$. Let $S$ be the set of all subsets of $H$ with an even number of elements; i.e.

$$
S=\{X: X \subset H,|X| \equiv 0 \bmod 2\}
$$

Let $\omega: S \rightarrow S$ denote set complementation in $H$. Then $S$ is partially ordered by set inclusion and orthocomplemented by $\omega$, with zero the empty set $\square$, and unit the set $H$. It is easy to see that $S$ is orthomodular, atomic and completely orthomodular.

$S$ is not a lattice because, for example, the pair of elements of $S$, $\{1,2\}$ and $\{2,3\}$, have no l.u.b. So by Proposition 4.1 of Finch [1], $S$ does not possess the $A$-property. This can be seen directly. Let $a_{1}=\{1,2\}$, $a_{2}=\{2,3\}$ and $b=\{4,5\}$. Then $a_{1} \wedge a_{2}=\square$ and $a_{i} \perp b(i=1,2)$. Also $a_{1} \vee b=\{1,2,4,5\}$ and $a_{2} \vee b=\{2,3,4,5\}$. Then $a_{1} \vee b$ and $a_{2} \vee b$ have no g.l.b. There are three maximal lower bounds; $\{2,4\},\{2,5\}$ and $\{4,5\}$.

Now consider the subset $X$ of $S$ defined by

Then

$$
X=\{\square,\{1,2\},\{2,3\},\{1,3\}\}
$$

and

$$
X \tau=\{a: a \in S,\{1,2,3\} \subset a\}
$$

$$
\begin{aligned}
X \tau \sigma & =\{a: a \in S, a \subset\{1,2,3\}\} \\
& =X .
\end{aligned}
$$


Now let $M=\{\{1,2\}\} . M$ is a maximal orthogonal subset of $X=X \tau \sigma$ but

$$
\begin{aligned}
M \tau \sigma & =\{\square,\{1,2\}\} \\
& \neq X \tau \sigma .
\end{aligned}
$$

Notice that the other maximal orthogonal subsets of $X$ are just $\{\{\mathbf{1}, \mathbf{3}\}\}$ and $\{\{2,3\}\}$; of the same form as $M$.

Thus $S$ is an orthomodular poset that does not have the $B$-property and so, by Proposition 3.2 of Finch [1], its completion by cuts is not orthomodular. This can be seen directly if we let $Y$ be the element of the completion by cuts of $S$ generated by the element $\{1,2,3,4\}$ of $S$. Then $X$, as defined above, is a proper subelement of $Y$. One can readily show that $Y \wedge X \Omega=\{\square\}$, and so the completion by cuts of $S$ is not orthomodular.

\section{Reference}

[1] Finch P. D., 'On Orthomodular Posets.' Preceding paper in this issue.

Monash University

Melbourne 